\title{
Testing the gaugino AMSB model at the Tevatron via slepton pair production
}

\author{
Howard Baer讯, Senarath de Alwis讯, Kevin Givens理,

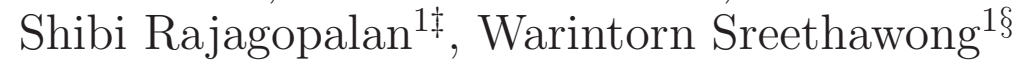

1. Dept. of Physics and Astronomy, University of Oklahoma, Norman, OK 73019, USA

2. Dept. of Physics, University of Colorado, Boulder CO 80309, USA

\begin{abstract}
Gaugino AMSB models- wherein scalar and trilinear soft SUSY breaking terms are suppressed at the GUT scale while gaugino masses adopt the AMSB form- yield a characteristic SUSY particle mass spectrum with light sleptons along with a nearly degenerate wino-like lightest neutralino and quasi-stable chargino. The left- sleptons and sneutrinos can be pair produced at sufficiently high rates to yield observable signals at the Fermilab Tevatron. We calculate the rate for isolated single and dilepton plus missing energy signals, along with the presence of one or two highly ionizing chargino tracks. We find that Tevatron experiments should be able to probe gravitino masses into the $\sim 55 \mathrm{TeV}$ range for inoAMSB models, which corresponds to a reach in gluino mass of over $1100 \mathrm{GeV}$.
\end{abstract}

PACS numbers: 14.80.Ly, 12.60.Jv, 11.30.Pb

\footnotetext{
*Email: baer@nhn.ou.edu

${ }^{\dagger}$ Email: dealwiss@colorado.edu

†Email: kevin.givens@colorado.edu

${ }^{\ddagger}$ Email: shibi@nhn.ou.edu

ミEmail: wstan@nhn.ou.edu
} 


\section{Introduction}

Searches for supersymmetry (SUSY) at the Fermilab Tevatron collider usually focus on gluino and squark pair production reactions, due to their large strong interaction production rates [1] 3], or on observation of chargino-neutralino production and decay to isolated trileptons, due to their low background rates [4] 6 . The possibility of observation of slepton pair production at the Tevatron was examined in Ref. [7] in the context of the MSSM with gaugino mass unification and found to be difficult: the dilepton signature from $p \bar{p} \rightarrow \tilde{\ell}^{+} \tilde{\ell}^{-} \rightarrow \ell^{+} \ell^{-}+E_{T}$ (here, $\ell=e$ or $\mu$ ) is beset with large backgrounds from $W^{+} W^{-}$and $Z \rightarrow \tau^{+} \tau^{-}$production, while the $\tilde{\ell} \tilde{\nu}_{L} \rightarrow \ell^{ \pm}+\mathbb{E}_{T}$ signal is beset by even larger backgrounds from direct $W^{ \pm} \rightarrow \ell^{ \pm} \nu_{\ell}$ production. However, these past works did not anticipate the Tevatron reaching integrated luminosities in the vicinity of $8-16 \mathrm{fb}^{-1}$.

In this paper, we investigate the recently introduced gaugino $A M S B$ model (inoAMSB) [8], which arises naturally from some highly motivated string theory constructions. The inoAMSB model gives rise to a characteristic SUSY particle mass spectrum which features 1. a winolike lightest neutralino $\widetilde{Z}_{1}, 2$. a nearly mass degenerate quasi-stable chargino $\widetilde{W}_{1}$, (points 1 and 2 also occur in previous AMSB constructs [9 11]), 3. a rather light spectrum of sleptons, arranged in a mass hierarchy $m_{\tilde{\nu}_{L}}<m_{\tilde{\ell}_{L}}<m_{\tilde{\ell}_{R}}$ and 4 . a rather heavy spectrum of squarks and gluinos, where $m_{\tilde{g}} \sim m_{\tilde{q}} \sim 7.5 m_{\widetilde{W}_{1}}$. Given the LEP2 limit on quasi-stable charginos from AMSB models, where $m_{\widetilde{W}_{1}}>91.9 \mathrm{GeV}\left[12\right.$, this implies $m_{\tilde{g}} \gtrsim 700 \mathrm{GeV}:$ quite beyond the reach of Tevatron. However, in inoAMSB models the sleptons can exist with masses as low as $\sim 130$ $\mathrm{GeV}$. Pair production of inoAMSB sleptons, followed by decays into quasi-stable charginos, should give rise to characteristic isolated single or dilepton plus $\mathbb{E}_{T}$ signatures, accompanied by the presence of one or two highly ionizing chargino tracks (HITs) [10].

In a previous work [8], we presented the spectrum of SUSY particle masses which are expected from inoAMSB models, and evaluated prospects for detection at the LHC with $\sqrt{s}=$ $14 \mathrm{TeV}$. A $100 \mathrm{fb}^{-1} \mathrm{LHC}$ reach to $m_{\tilde{g}} \sim 2.3 \mathrm{TeV}$ was found. The gluino and squark cascade decay [13] events would often contain the presence of highly ionizing chargino tracks that could range up to a few $\mathrm{cm}$ in length. The unique inoAMSB mass spectrum $m_{\widetilde{Z}_{2}}>m_{\tilde{\ell}_{R}}>m_{\tilde{\ell}_{L}}>$ $m_{\widetilde{W}_{1}, \widetilde{Z}_{1}}$ leads to a characteristic double bump (mass edge) structure in the opposite-sign dilepton invariant mass distribution which could serve to distinguish the inoAMSB model from minimal AMSB (mAMSB) or hypercharged AMSB [14] (HCAMSB).

In Ref. [15, the relic density of dark matter in inoAMSB (and also in mAMSB and HCAMSB) was considered. In all AMSB models with sub-TeV scale $\widetilde{Z}_{1}$, the thermal abundance of neutralino cold dark matter is well below the WMAP-measured value of $\Omega_{C D M} h^{2}=0.1123 \pm 0.0035$ [16]. However, the possibility of additional neutralino production via moduli [17], gravitino [18] or axino [19] decay can augment the thermal abundance, bringing the expected neutralino abundance into accord with measured values.

In this paper, we calculate signal rates for slepton pair production in inoAMSB models at the Fermilab Tevatron collider. We find a considerable reach for the nearly background free signature of single or OS dilepton plus $E_{T}$ plus one or two HITs [10]; these signal rates ought to allow Tevatron experiments to explore slepton masses from the inoAMSB model into the 200 $\mathrm{GeV}$ range for $\sim 10 \mathrm{fb}^{-1}$ of integrated luminosity, corresponding to a reach in $m_{3 / 2}$ of over 50 $\mathrm{TeV}$. 


\section{The gaugino AMSB model}

Gaugino Anomaly Mediated Supersymmetry Breaking [8] is a very simple scenario for generating SUSY breaking soft terms in low energy supersymmetric theories. The main assumption is that the high energy theory which generates SUSY breaking is of the sequestered type [9], which effectively means that the classical gaugino and scalar masses and $A$-terms are highly suppressed relative to the gravitino mass scale. This is in contrast to the situation in usual supergravity (SUGRA) models, where these soft parameters are classically generated at the gravitino mass scale. Nevertheless, in contrast to what is usually advocated in AMSB [9], it has been argued [20] that only gaugino masses are generated by Weyl anomalies. In inoAMSB [8] 21], the scalar masses are then generated by renormalization group (RG) running as in what is often called gaugino mediation [22] or simple no-scale SUSY breaking models [23]. The inoAMSB model then avoids both the generic FCNC problems of gravity mediated scenarios and also the tachyonic slepton problem of the traditional AMSB construct. It also avoids the presence of tau slepton LSPs which occur in gaugino mediation/no-scale models with gaugino masses unified at a high scale.

This very simple phenomenological model depends on just two parameters: the gravitino mass $m_{3 / 2}$ which sets the scale for all sparticle masses, and $\tan \beta$, the ratio of the the Higgs vacuum expectation values in the MSSM. In fact, it appears to be the simplest SUSY mediation model that one can conceive of which satisfies all phenomenological constraints.

Furthermore, inoAMSB can be realized within a highly motivated class of string theories [21]. The models in question are called the large volume compactification scenario (LVS) of type IIB string theory and were introduced in [24. The moduli (and the dilaton) of string theory, which appear as 4D fields in the effective action, are stabilized using a combination of fluxes and non-perturbative effects (for reviews see [25]). The Calabi-Yau (CY) manifolds on which the theory is compactified to 4D is of the so-called "Swiss Cheese" type with one large four cycle (which controls the overall size of the internal space) and one or more small cycle. An analysis of the potential for the moduli shows that the volume is exponentially large in the small cycle(s) whose size in turn is stabilized at values larger than the string scale. The effective parameter which controls this is determined by the Euler character of the CY manifold and the (flux dependent) value of the dilaton.

It was shown in 21] that in these models, for large enough volume (greater than $10^{5}$ Planck units), FCNC effects are suppressed. Indeed, all classically generated soft SUSY breaking parameters are volume suppressed compared to the gaugino mass soft terms that are generated by anomaly mediation. The latter effect is actually a consequence of the generation of gaugino masses by the Weyl anomaly effect as discussed in [20].

The phenomenology of this class of string theoretic models is effectively controlled by the gravitino mass. But the theory at this point only allows us to estimate an upper bound to the possible size of $\mu$ and $B$ terms. So we use the latter after trading it for (as is usual) $\tan \beta$, and regard the former as an output from the experimental value of the $Z$ mass. The parameters of the phenomenological model which comes from these string theory considerations are thus

$$
m_{3 / 2}, \tan \beta, \operatorname{sign}(\mu) .
$$

The gravitino mass determines the values of the gaugino masses at the high scale (which will 
be chosen to be the GUT scale) by the Weyl anomaly formula given in [20]. It turns out that for the scenario in [21], this is exactly the same as what is often given as the AMSB formula for these masses i.e.

$$
M_{i}=\frac{b_{i} g_{i}^{2}}{16 \pi^{2}} m_{3 / 2}
$$

with $b_{i}=(33 / 5,1,-3)$. The initial (high scale) values of the other soft parameters are then taken to be

$$
m_{0}=A_{0}=0,
$$

where $m_{0}$ is the common soft SUSY breaking scalar mass evaluated at the high scale $\sim M_{\text {string }}$ or $M_{G U T}$, and $A_{0}$ is the trilinear soft SUSY breaking (SSB) term.

\section{Production and decay of inoAMSB sleptons at the Tevatron}

We begin by examining the sort of sparticle mass spectra that is expected from the inoAMSB boundary conditions: $m_{0}=A_{0}=0$ but with $M_{i}=\frac{b_{i} g_{i}^{2}}{16 \pi^{2}} m_{3 / 2}$. We adopt a unified value of the gauge coupling $g_{G U T}=0.714$ and then for a given value of $m_{3 / 2}$ compute the GUT scale values of the three gaugino masses $M_{i}$ for $i=1-3$. We compute the sparticle mass spectra using the Isasugra subprogram of the event generator Isajet [26], along with the option of non-universal gaugino masses. The parameter space is that of Eq. 1.

After input of the above parameter set, Isasugra implements an iterative procedure of solving the MSSM RGEs for the 26 coupled renormalization group equations, taking the weak scale measured gauge couplings and third generation Yukawa couplings as inputs, as well as the above-listed GUT scale SSB terms. Isasugra implements full 2-loop RG running in the $\overline{D R}$ scheme, and minimizes the RG-improved 1-loop effective potential at an optimized scale choice $Q=\sqrt{m_{\tilde{t}_{L}} m_{\tilde{t}_{R}}}$ (which accounts for leading two-loop terms) [27] to determine the magnitude of $\mu$ and the value of $m_{A}$. All physical sparticle masses are computed with complete 1-loop corrections, and 1-loop weak scale threshold corrections are implemented for the $t, b$ and $\tau$ Yukawa couplings [28]. The off-set of the weak scale boundary conditions due to threshold corrections (which depend on the entire superparticle mass spectrum), necessitates an iterative up-down RG running solution. The resulting superparticle mass spectrum is typically in close accord with other sparticle spectrum generators [29].

In Fig. 1, we show the mass spectrum of various sleptons and light gauginos of interest to Tevatron experiments versus $m_{3 / 2}$ for $\tan \beta=10$ and $\mu>0$. Results hardly change if we flip the sign of $\mu$. If $\tan \beta$ is increased, then third generation squark and slepton and heavy Higgs masses decrease, while first/second generation slepton masses of interest here remain nearly the same. We see from Fig. 1 that while charginos and neutralinos are predicted to be the lightest MSSM particles, $\tilde{\ell}_{L}$ and $\tilde{\nu}_{L}$ are also quite light- as low as $\sim 130 \mathrm{GeV}$ - with $m_{\tilde{\nu}_{L}}<m_{\tilde{\ell}_{L}}$. Unlike mSUGRA or mAMSB, the $\tilde{\ell}_{R}$ mass is split from $\tilde{\ell}_{L}$ and quite a bit heavier: at least $280 \mathrm{GeV}$ in inoAMSB. The $\widetilde{Z}_{2}$ is bino-like, with $m_{\widetilde{Z}_{2}}>m_{\tilde{\ell}_{R}}$.

In Fig. 2, we show various slepton pair production cross sections as calculated at NLO [30] 


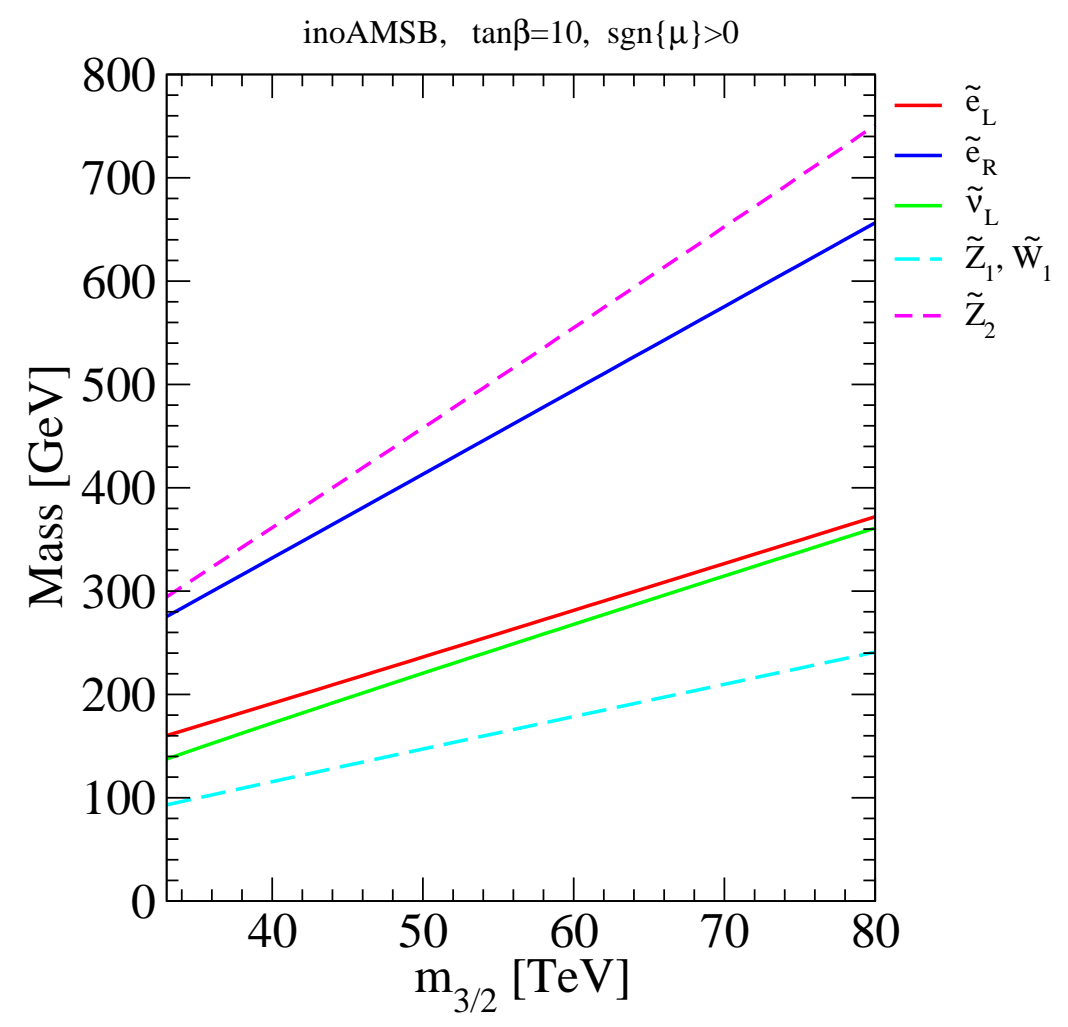

Figure 1: Plot of various gaugino and slepton masses in the inoAMSB model versus $m_{3 / 2}$ for $\tan \beta=10$ and $\mu>0$.

using the Prospino program [31, 32, 1 The results are calculated versus $m_{3 / 2}$ for the same parameters as in Fig. 1. We do not present $\widetilde{W}_{1} \widetilde{W}_{1}$ or $\widetilde{W}_{1} \widetilde{Z}_{1}$ cross sections, since the visible energy from quasi-stable $\widetilde{W}_{1} \rightarrow \pi \widetilde{Z}_{1}$ decay is insufficient to trigger on.

From Fig. 2, we see that the reactions $p \bar{p} \rightarrow \tilde{e}_{L}^{ \pm} \tilde{\nu}_{e L}, p \bar{p} \rightarrow \tilde{\nu}_{e L} \overline{\tilde{\nu}}_{e L}$ and $p \bar{p} \rightarrow \tilde{e}_{L} \overline{\tilde{e}}_{L}$ are comparable and can exceed the $1 \mathrm{fb}$ level for $m_{3 / 2} \lesssim 45 \mathrm{GeV}$. They reach a maximum value of $\sim 10 \mathrm{fb}$ for $m_{3 / 2} \sim 33 \mathrm{TeV}$. When we sum over $\ell=e, \mu$ and $\tau$, then the total slepton pair production is even larger. The $\tilde{e}_{R} \overline{\tilde{e}}_{R}$ pair production is much lower in rate, and unobservable at projected Tevatron luminosities. Also, we see that cross sections involving $\widetilde{Z}_{2}$ production are much smaller, and won't contribute to the observable rates. For $m_{3 / 2} \gtrsim 60 \mathrm{TeV}$, the slepton pair production cross sections drop below the $0.1 \mathrm{fb}$ level, and are likely unobservable at Tevatron.

To determine the slepton pair producton signatures, we must next calculate their branching fractions [34]. Using Isajet, we find the following values:

- $\tilde{\nu}_{\ell L} \rightarrow \widetilde{Z}_{1} \nu_{\ell} 33 \%$,

\footnotetext{
${ }^{1}$ Recent works on slepton pair production at hadron colliders including resummation effects are included in Ref. 33.
} 


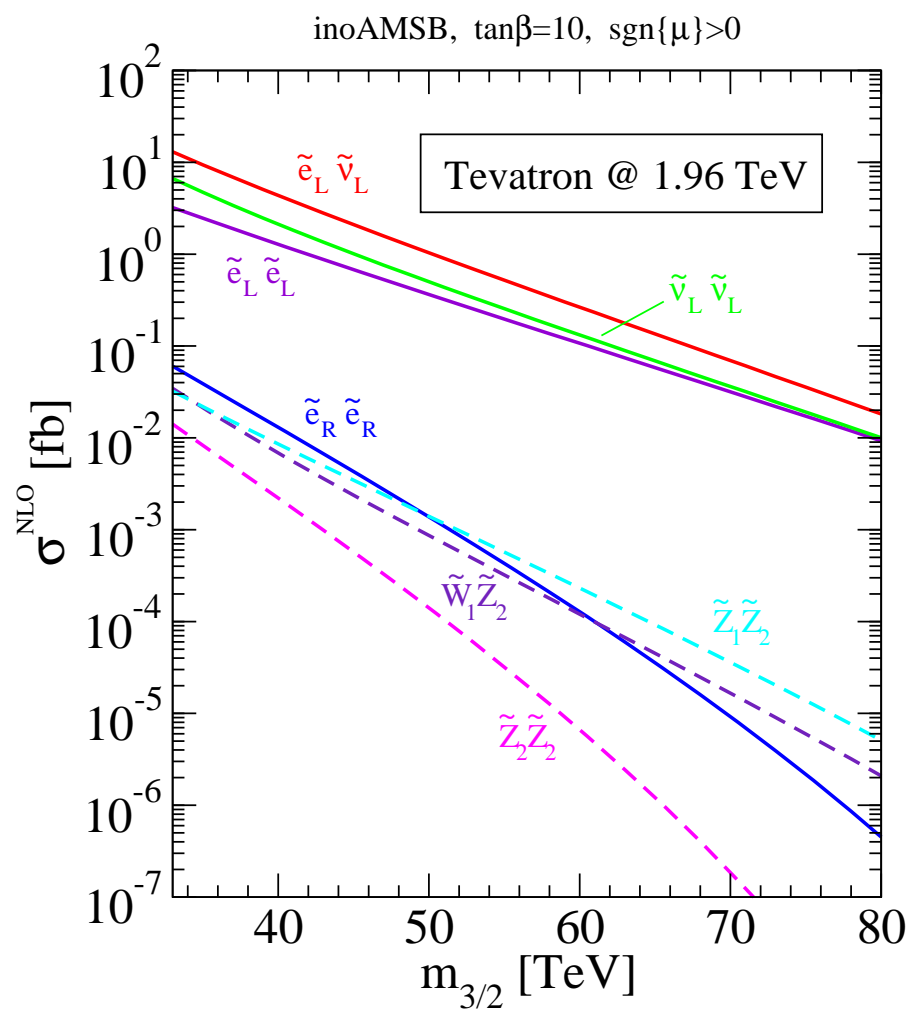

Figure 2: Plot of various slepton and gaugino pair production cross sections at the Fermilab Tevatron collider with $\sqrt{s}=1.96 \mathrm{TeV}$ for the inoAMSB model. We plot versus $m_{3 / 2}$ for $\tan \beta=10$ and $\mu>0$.

- $\tilde{\nu}_{\ell L} \rightarrow \widetilde{W}_{1} \ell \quad 67 \%$,

while

- $\tilde{\ell}_{L} \rightarrow \widetilde{Z}_{1} \ell \quad 33 \%$,

- $\tilde{\ell}_{L} \rightarrow \widetilde{W}_{1} \nu_{\ell} \quad 67 \%$.

Each quasi-stable chargino gives rise to a HIT, which may be visible in the microvertex tracker. By combining branching fractions with slepton pair production, we find the following event topologies.

1. $\tilde{\ell}_{L} \tilde{\nu}_{\ell L} \rightarrow \ell+2 \operatorname{HIT} s+E_{T} \quad 45 \%$,

2. $\tilde{\ell}_{L} \tilde{\nu}_{\ell L} \rightarrow \ell^{+} \ell^{-}+H I T+E_{T}, 22 \%$,

3. $\tilde{\ell}_{L} \tilde{\nu}_{\ell L} \rightarrow \ell+E_{T}, \quad 10 \%$,

4. $\tilde{\nu}_{\ell L} \overline{\tilde{\nu}}_{\ell L} \rightarrow \ell^{+} \ell^{-}+2$ HITs $+\#_{T}, \quad 45 \%$, 
5. $\tilde{\nu}_{\ell L} \overline{\tilde{\nu}}_{\ell L} \rightarrow \ell+H I T+E_{T}, \quad 44 \%$,

6. $\tilde{\ell}_{L} \overline{\tilde{\ell}}_{L} \rightarrow \ell+H I T+E_{T}, \quad 44 \%$,

7. $\tilde{\ell}_{L} \overline{\tilde{\ell}}_{L} \rightarrow \ell^{+} \ell^{-}+E_{T}, \quad 10 \%$.

The $\ell^{+} \ell^{-}+E_{T}$ topology from reaction 7 will likely be swamped by $W W$ and $Z \rightarrow \tau^{+} \tau^{-}$backgrounds, while the $\ell^{ \pm}+E_{T}$ topology from reaction 3 will be buried under $W \rightarrow \ell \nu_{\ell}$ background. However, the event topologies including HITs should stand out from SM background, and furthermore, should signal the presence of the quasi-stable chargino. We note here that topologies 1 and 2 are unique to $\tilde{\ell}_{L} \tilde{\nu}_{L}$ production, while topology 4 is unique to $\tilde{\nu}_{\ell L} \overline{\tilde{\nu}}_{\ell L}$ production. If a two HIT topology has one of the HITs missed for some reason, it will look like a single HIT event. But the 2 HIT topologies 1 and 4 are unique in that they each contain two quasi-stable chargino tracks. Thus, these topologies will pinpoint the particular superparticle production mechanism. Topologies 5 and 6 arise from both $\tilde{\nu}_{L} \overline{\tilde{\nu}}_{L}$ and $\tilde{\ell}_{L} \overline{\tilde{\ell}}_{L}$ production.

The first/second generation slepton masses and branching fractions listed above are largely immune to variations in $\tan \beta$, so even if $\tan \beta$ changes over the range $\sim 5-40$ (parameter space maxes out at $\tan \beta \sim 42$; see Fig. 5 of Ref. 8), the expected signatures are expected to be nearly $\tan \beta$ invariant. As $\tan \beta$ increases, the $\tilde{\tau}_{1}$ and $\tilde{\nu}_{\tau L}$ masses decrease, leading to a somewhat increased rate for production of one of two tau leptons plus HITs plus $\mathbb{E}_{T}$ relative to production of one or two isolated $\ell$ s plus HITs plus $E_{T}$.

\section{Signal and background after cuts}

Once the superparticle mass spectrum and decay branching fractions have been calculated using Isasugra, the output is fed into Herwig [35] for event generation using $p \bar{p}$ collisions at $\sqrt{s}=1.96$ $\mathrm{TeV}$. We adopt the AcerDet toy detector simulation program as well [36]. We then generate all superparticle production events. A large component from $\widetilde{W}_{1} \widetilde{Z}_{1}$ and $\widetilde{W}_{1}^{+} \widetilde{W}_{1}^{-}$production will not provide enough visible energy for triggers, so we focus instead on slepton pair production, where the signal is an opposite-sign/same flavor (OSSF) dilepton pair $\left(e^{+} e^{-}\right.$or $\left.\mu^{+} \mu^{-}\right)$plus missing $E_{T}$ (MET).

To gain perspective on the energy scales from slepton pair production, we plot first in Fig. 3 the $p_{T}$ distribution of the hardest $\left(\ell_{1}\right)$ and softest $\left(\ell_{2}\right)$ leptons from slepton pair production in inoAMSB with $m_{3 / 2}=35 \mathrm{TeV}, \tan \beta=10$ and $\mu>0$. So far, we have imposed no cuts, so the events come from pure slepton pair production with either one or two isolated leptons in the final state. The $p_{T}\left(\ell_{1}\right)$ distribution spans an approximate range $\sim 30-120 \mathrm{GeV}$, with a peak at $\sim 65 \mathrm{GeV}$. The second lepton $p_{T}$ distribution spans $\sim 10-80 \mathrm{GeV}$, with a peak at $\sim 20 \mathrm{GeV}$. We also show the expected MET distribution, which peaks around $60 \mathrm{GeV}$.

In Fig. 4 , we show the OSSF dilepton opening angle in the transverse plane. The distribution peaks around $\Delta \phi\left(\ell^{+} \ell^{-}\right) \sim \pi$, reflecting the fact that the sleptons are produced back-to-back in the transverse direction. However, when the lepton momentum from slepton decay is boosted to the LAB frame, the distribution smears out considerably: while most events occur at large transverse opening angle, there is a significant probability for both detected leptons to appear on the same side of the detector, i.e. with $\Delta \phi\left(\ell^{+} \ell^{-}\right)<\pi / 2$. 


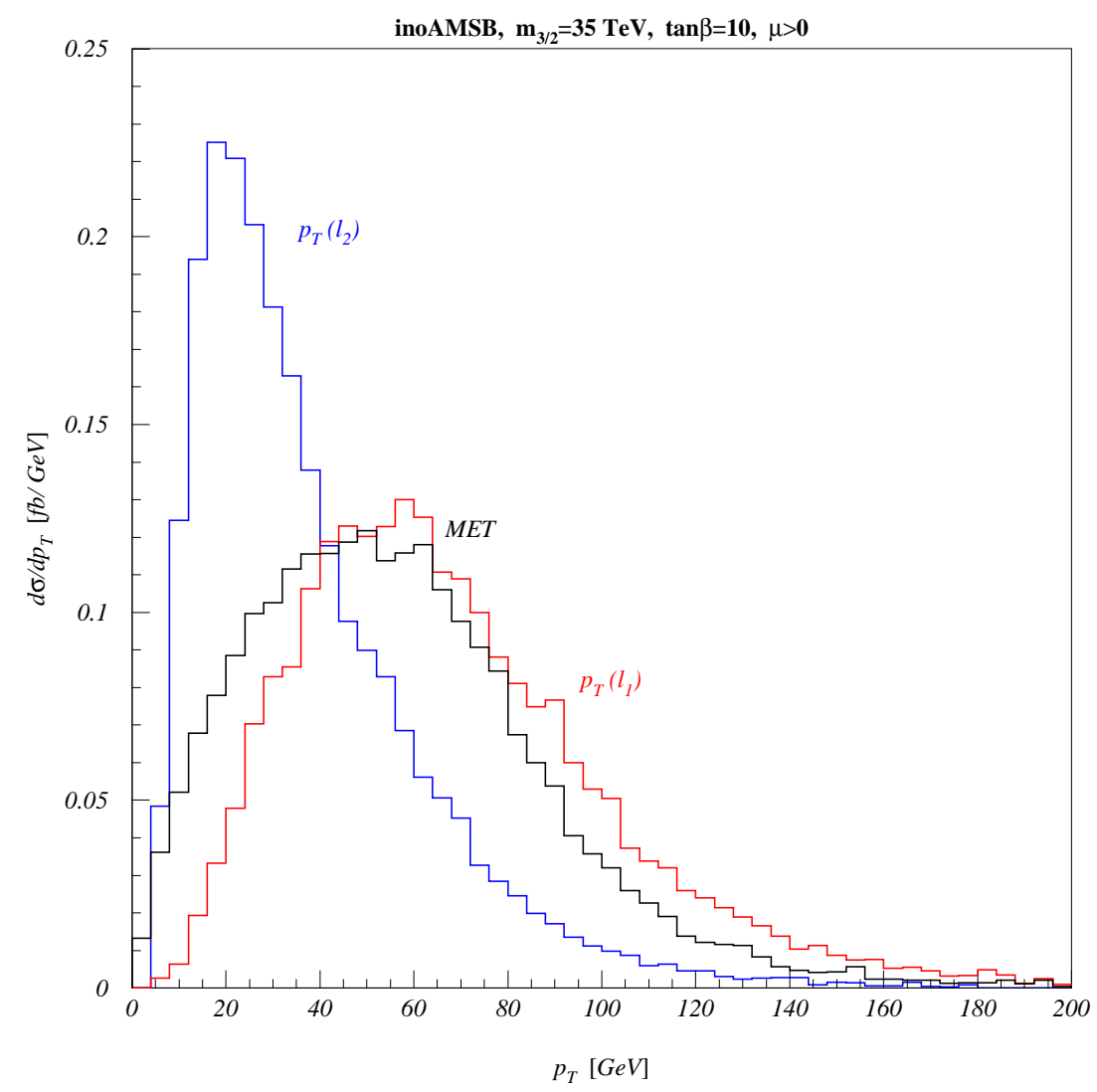

Figure 3: Plot of $p_{T}$ distribution of hardest lepton, second hardest lepton and MET from slepton pair production events with OSSF dileptons at the Fermilab Tevatron for the inoAMSB model. We adopt $m_{3 / 2}=35 \mathrm{TeV}, \tan \beta=10$ and $\mu>0$.

Following recent CDF/D0 analyses of $W$ and $Z$ production [37, 38, we next impose a minimal set of cuts:

- $E_{T}>25 \mathrm{GeV}$,

- at least one isolated lepton $\left(e\right.$ or $\mu$ ) with $p_{T}(\ell)>25 \mathrm{GeV}$ and $|\eta(\ell)|<1$,

- for two lepton events, $p_{T}\left(\ell_{2}\right)>25 \mathrm{GeV}$ and $\left|\eta\left(\ell_{2}\right)\right|<2$,

- for events containing HITs, we require $|\eta(H I T)|<2$.

Next, keeping $\tan \beta=10$ and $\mu>0$, we scan over $m_{3 / 2}$ values from $30-80 \mathrm{TeV}$. The rates for various single and OSSF dilepton events, with 0,1 , or 2 HITs, are shown in Fig. 5. We also compute single and OSSF dilepton background rates from $p \bar{p} \rightarrow W^{ \pm} \rightarrow \ell \nu_{\ell}$ production, and $W^{+} W^{-}$and $Z \rightarrow \tau^{+} \tau^{-}$production, respectively. The single lepton background from $W$ production is about six orders of magnitude above signal, making a search in this channel 


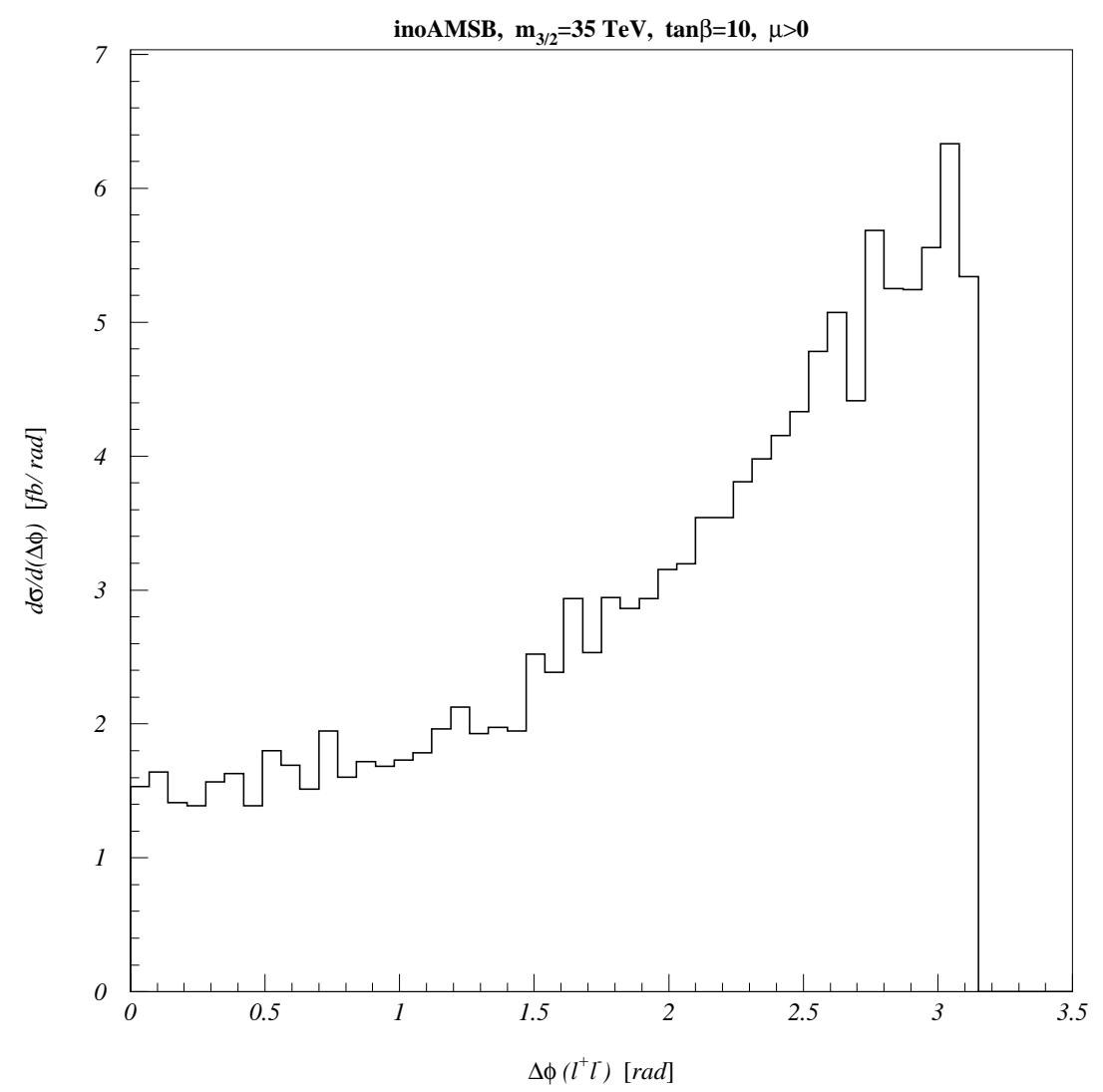

Figure 4: Distribution in OSSF dilepton transverse opening angle at the Fermilab Tevatron for the inoAMSB model. We adopt $m_{3 / 2}=35 \mathrm{TeV}, \tan \beta=10$ and $\mu>0$.

hopeless. The $W W$ and $\tau^{+} \tau^{-}$backgrounds are somewhat above the largest OSSF dilepton signal levels.

At this stage, it is important to note that most signal events will contain at least one HIT, which should be well separated in angle from the isolated leptons. The presence of HITs should allow distinguishability of signal from background. The efficiency for HIT identification is detector dependent, and beyond the scope of our theory analysis: here we will assume a HIT identification efficiency of $100 \%$. Long-lived tracks from hyperon production with $\Xi \rightarrow \Lambda \pi$ decay have been identified by the CDF collaboration in the SVX detector and used to great effect in their analysis of $\Xi_{b}$ production and decay [39]. If we require the presence of one or more HITs from quasi-stable charginos, then SM background should be largely negligible. In particular, the $\ell^{+} \ell^{-}+2 H I T s+E_{T}$ signal from sneutrino pair production followed by $\tilde{\nu}_{\ell} \rightarrow \ell \widetilde{W}_{1}$ decay should provide a smoking gun signature for inoAMSB at the Tevatron. From Fig. 5, we see that this cross section ranges up to $2 \mathrm{fb}$ after cuts. With $\sim 10 \mathrm{fb}^{-1}$ of integrated luminosity, Tevatron experiments may have a reach for the inoAMSB model in this channel to $m_{3 / 2} \sim 40-50 \mathrm{TeV}$. The $1 \ell+2$ HITs $+\mathbb{E}_{T}$ channel, coming from $\tilde{e}_{L} \tilde{\nu}_{\ell L}$ production, is generically about a factor 3 higher than the $\ell^{+} \ell^{-}+2 H I T s+E_{T}$ channel, and should provide 


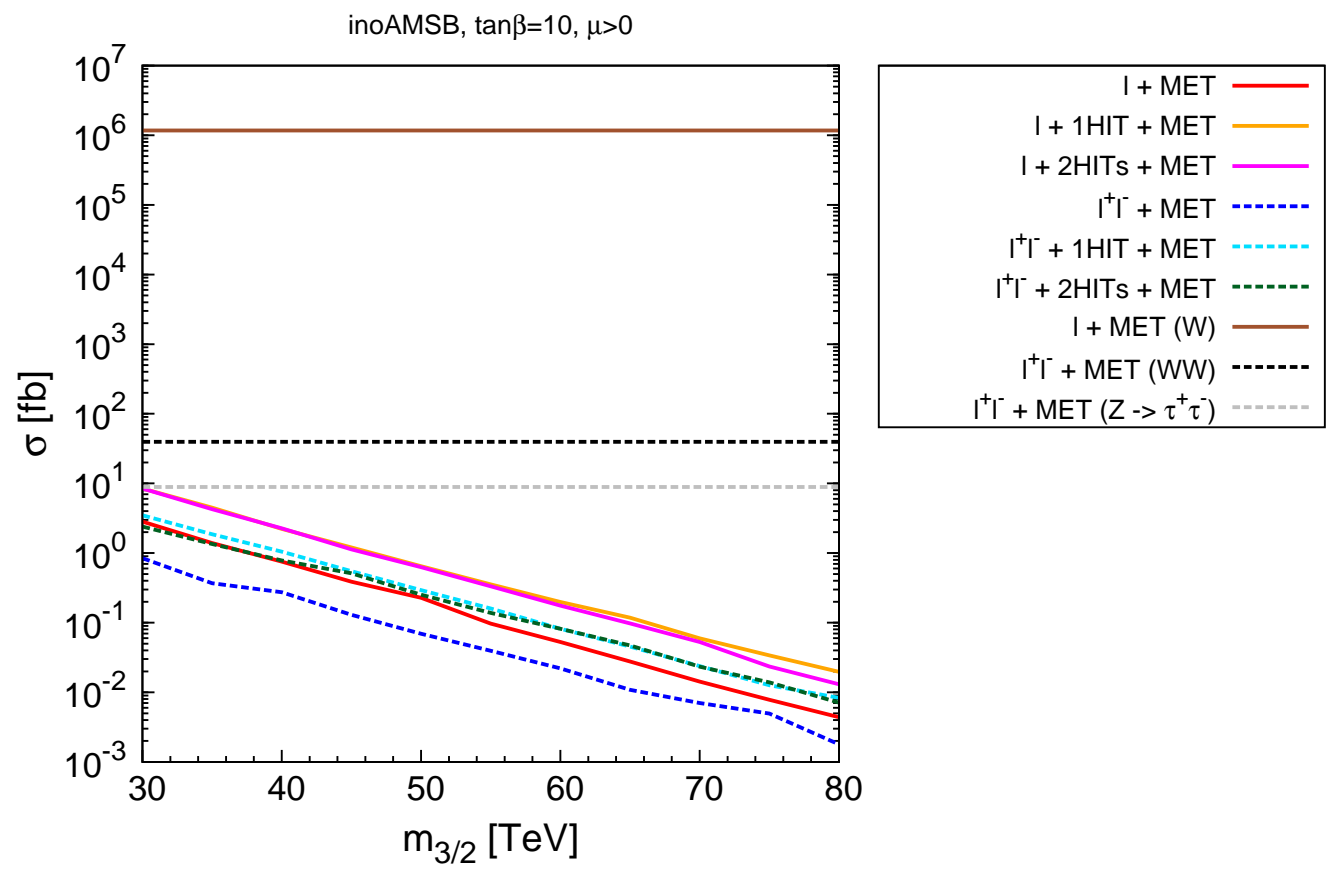

Figure 5: Plot of various isolated lepton plus $\mathbb{E}_{T}$ event topologies after cuts at the Fermilab Tevatron for the ino $A M S B$ model. Here, $\ell=e$ or $\mu$. We plot signal rate after cuts versus $m_{3 / 2}$ for $\tan \beta=10$ and $\mu>0$.

corroborating evidence. There are also comparable contributions to the $\ell+H I T+\mathbb{E}_{T}$ and $\ell^{+} \ell^{-}+H I T+E_{T}$ channels. By combining all channels, the $10 \mathrm{fb}^{-1}$ reach of Tevatron for slepton pair production in inoAMSB models should extend to $m_{3 / 2} \sim 55 \mathrm{GeV}$. Augmenting the signal with single tau-jet and ditau-jets plus $H I T s+\mathbb{E}_{T}$ events will increase the reach even further.

Once an inoAMSB signal for slepton pair production is established, then the next step will be to try to extract sparticle masses from the event kinematics. We will first look at the sneutrino pair production reaction $p \bar{p} \rightarrow \tilde{\nu}_{\ell L} \bar{\nu}_{\ell L} \rightarrow \ell^{+} \ell^{-}+2$ HITs $+E_{T}$, which arises when $\tilde{\nu}_{\ell L} \rightarrow \ell \widetilde{W}_{1}$ decay. Since the $\widetilde{W}_{1}$ gives essentially all $\not_{T^{-}}$aside from the HIT-it would be useful to construct the transverse mass [40] from the $\tilde{\nu}_{\ell L}$ decay:

$$
m_{T}^{2}\left(\ell, \vec{B}_{T}\right)=\left(\left|\vec{p}_{\ell T}\right|+\left|\vec{B}_{T}\right|\right)^{2}-\left(\vec{p}_{\ell T}+\vec{\not}_{T}\right)^{2}
$$

from each signal event, since this quantity is bounded by $m_{T}(\max )=m_{\tilde{\nu}_{\ell L}}\left(1-m_{\widetilde{W}_{1}}^{2} / m_{\tilde{\nu}_{\ell L}}^{2}\right)$. However, since we do not a priori know the value of $p_{T}\left(\widetilde{W}_{1}\right)$, but only know $\vec{E}_{T} \simeq \vec{p}_{T}\left(\widetilde{W}_{1}\right)+$ $\vec{p}_{T}\left(\widetilde{W}_{1}^{\prime}\right)$, we must instead use the Cambridge $m_{T 2}$ variable [41]:

$$
m_{T 2}=\underset{\vec{p}_{T}\left(\widetilde{W}_{1}\right)=\vec{H}_{T}-\vec{p}_{T}\left(\widetilde{W}_{1}^{\prime}\right)}{ }\left[\max \left(m_{T}\left(\ell_{1}, \vec{p}_{T}\left(\widetilde{W}_{1}\right)\right), m_{T}\left(\ell_{2}, \vec{p}_{T}\left(\widetilde{W}_{1}^{\prime}\right)\right)\right)\right]
$$

which by construction must be bounded by the $m_{T}$ value which is constructed with the correct lepton and missing $E_{T}$ vectors. 


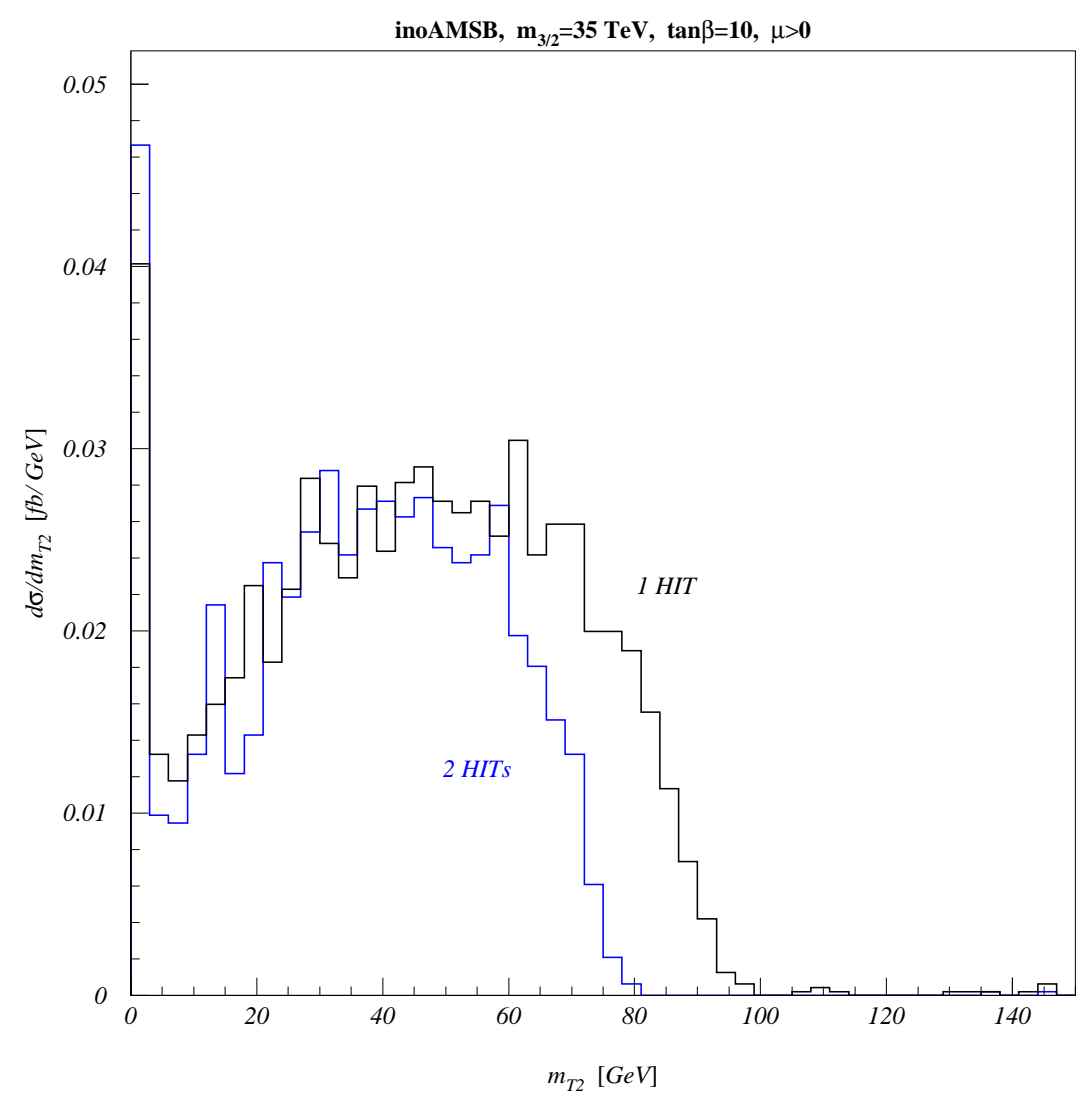

Figure 6: Distribution in variable $m_{T 2}\left(\ell^{+}, \ell^{-}, E_{T}\right)$ from OSSF slepton pair events at the Tevatron for events containing 1 or 2 HITs. We plot for $m_{3 / 2}=35 \mathrm{TeV}, \tan \beta=10$ and $\mu>0$.

The distribution in $m_{T 2}$ for $\ell^{+} \ell^{-}+2 H I T s+E_{T}$ is shown as the blue histogram in Fig. 6. We see as expected a continuum distribution followed by a visible cut-off around $m_{T}(\max ) \simeq 73.4$ $\mathrm{GeV}$.

If instead we examine the $m_{T 2}$ distribution for $\ell^{+} \ell^{-}+1 H I T+\mathbb{E}_{T}$, then we will mainly pick up $\tilde{\ell}_{L}^{+} \tilde{\ell}_{L}^{-}$production, plus some fraction of $\tilde{\nu}_{\ell L} \overline{\tilde{\nu}}_{\ell L}$ events where one of the HITs is missed, perhaps due to having too high $|\eta|>2$ value. In this case, $m_{T 2}$ is bounded by $105.9 \mathrm{GeV}$, as is illustrated in Fig. 6,

\subsection{Slepton pair production in mAMSB}

We note here that Tevatron experiments can be sensitive to slepton pair production in the mAMSB model as well [10]. Light sleptons occur in mAMSB for very low values of the $m_{0}$ parameter. We have examined a case in the mAMSB model with $m_{0}=220 \mathrm{GeV}, m_{3 / 2}=35$ $\mathrm{TeV}, \tan \beta=10$ and $\mu>0$. This mAMSB benchmark gives rise to a spectrum with $m_{\tilde{\tau}_{1}}=124$ $\mathrm{GeV}, m_{\tilde{\nu}_{\ell L}}=150 \mathrm{GeV}, m_{\tilde{\ell}_{R}}=160.3 \mathrm{GeV}, m_{\tilde{\ell}_{L}}=174 \mathrm{GeV}$ and $m_{\widetilde{W}_{1}, \widetilde{Z}_{1}} \simeq 99.3 \mathrm{GeV}$. The event rates and distributions are rather similar to the inoAMSB model with $m_{3 / 2}=35 \mathrm{TeV}$. Naively, 
one might expect $\tau^{+} \tau^{-}+H I T s+\mathbb{E}_{T}$ production to occur at higher rates in mAMSB than in inoAMSB, since in mAMSB, the $\tilde{\tau}_{1}$ is NLSP, while in inoAMSB, the $\tilde{\nu}_{\ell L}$ is NLSP. However, since $m_{\tilde{\ell}_{R}}$ is quite a bit lighter in mAMSB than in inoAMSB, production of $\ell^{+} \ell^{-}+H I T s+E_{T}$ is augmented by $\tilde{\ell}_{R}^{+} \tilde{\ell}_{R}^{-}$production. Detailed simulations find a ratio

$$
R=\frac{N\left(\tau^{+} \tau^{-}+2 H I T s+E_{T}\right)}{N\left(\ell^{+} \ell^{-}+2 H I T s+E_{T}\right)}
$$

to be 0.16 for inoAMSB while $R=0.18$ for mAMSB (here, we require $p_{T}(\tau-j e t)>20 \mathrm{GeV}$ and $|\eta(\tau-j e t)|<2)$. Thus, it looks difficult to distinguish the two models at the Tevatron based on slepton pair production. Distinguishing the two models is straightforward once enough integrated luminosity is accumulated at LHC, since then $\widetilde{Z}_{2}$ s that are produced in gluino and squark cascade decays lead to a double edge structure in the $m\left(\ell^{+} \ell^{-}\right.$) distribution (reflecting the large $m_{\tilde{\ell}_{L}}, m_{\tilde{\ell}_{R}}$ mass gap) while the mAMSB model with light sleptons gives only a single mass edge, owing to the near degeneracy of $\tilde{\ell}_{R}$ and $\tilde{\ell}_{L}$ [8]. We also emphasize here that slepton pair production only occurs in mAMSB for very low $m_{0}$ and $m_{3 / 2}$ values, and the $m_{\tilde{\ell}_{L, R}}-m_{\widetilde{Z}_{1}}$ mass gap is quite variable for different $m_{0}$ values, while in inoAMSB, this mass gap is essentially a fixed prediction depending only on $m_{3 / 2}$.

\section{Conclusions}

In this paper, we have examined the possibility of detecting slepton pair production from the gaugino AMSB model at the Fermilab Tevatron, with $10-16 \mathrm{fb}^{-1}$ of integrated luminosity. This model is characterized by a spectrum of very light sleptons, along with a wino-like neutralino and a nearly mass degenerate, quasi-stable chargino; the latter occur in most AMSB-type models. In inoAMSB, the sneutrinos are the lightest sleptons, but they can decay visibly into modes such as $\tilde{\nu}_{\ell L} \rightarrow \ell \widetilde{W}_{1}$. If the highly ionizing chargino tracks (HITs) can be identified, then the $\ell+H I T s+E_{T}$ and $\ell^{+} \ell^{-}+H I T s+E_{T}$ signatures should be nearly background free. Summing over all production reactions and final states containing HITs should give the Fermilab Tevatron a reach in $m_{3 / 2}$ to $\sim 55 \mathrm{TeV}$, which corresponds to a gluino mass of $\sim 1200$ $\mathrm{GeV}$. This should be somewhat beyond what LHC can explore with $\sqrt{s}=7 \mathrm{TeV}$ and $\sim 1 \mathrm{fb}^{-1}$ of integrated luminosity [42]. If a sizable signal is established, then the distribution in $m_{T 2}$ should provide some information on the masses of the sparticles being produced. In particular, the max of the $m_{T 2}$ distribution should be somewhat higher for dilepton events with one HIT, as opposed to dilepton events containing two HITs. This reflects the $m_{\tilde{\ell}_{L}}>m_{\tilde{\nu}_{\ell L}}$ mass hierarchy which is expected from inoAMSB models.

\section{Acknowledgments}

We thank Yuri Gershtein for discussions. This work was supported in part by the U.S. Department of Energy. SdA and KG are supported in part by the United States Department of Energy under grant DE-FG02-91-ER-40672. 


\section{References}

[1] H. Baer and E. Berger, Phys. Rev. D 34 (1986) 1361; H. Baer, X. Tata and J. Woodside, Phys. Rev. D 41 (1990) 906; H. Baer, C. Kao and X. Tata, Phys. Rev. D 48 (1993) 2978; H. Baer, C. H. Chen, C. Kao and X. Tata, Phys. Rev. D 52 (1995) 1565; H. Baer, C. H. Chen, F. Paige and X. Tata, Phys. Rev. D 54 (1996) 5866; H. Baer, C. H. Chen, M. Drees, F. Paige and X. Tata, Phys. Rev. D 58 (1998) 075008; H. Baer, A. Mustafayev, S. Profumo and X. Tata, Phys. Rev. D 75 (2007) 035004; H. Baer, S. Kraml, A. Lessa, S. Sekmen and H. Summy, Phys. Lett. B 685 (2010) 72 .

[2] T. Aaltonen et al. (CDF Collaboration), Phys. Rev. Lett. 102 (2009) 121801.

[3] V. M. Abazov et al. (D0 Collaboration), Phys. Lett. B 660 (2008) 449.

[4] H. Baer and X. Tata, Phys. Rev. D 47 (1993) 2739; H. Baer, C. Kao and X. Tata, Phys. Rev. D 48 (1993) 5175; V. Barger, C. Kao and T. J. Li, Phys. Lett. B 433 (1998) 328; H. Baer, M. Drees, F. Paige, P. Quintana and X. Tata, Phys. Rev. D 61 (2000) 095007; V. Barger and C. Kao, Phys. Rev. D 60 (1999) 115015; K. Matchev and D. Pierce, Phys. Lett. B 467 (1999) 225; H. Baer, T. Krupovnickas and X. Tata, J. High Energy Phys. 0307 (2003) 020.

[5] T. Aaltonen et al. (CDF Collaboration), Phys. Rev. Lett. 101 (2008) 251801.

[6] V. M. Abazov et al. (D0 Collaboration), Phys. Lett. B 680 (2009) 34.

[7] H. Baer, C. H. Chen, F. Paige and X. Tata, Phys. Rev. D 49 (1994) 3283.

[8] H. Baer, S. de Alwis, K. Givens, S. Rajagopalan and H. Summy, JHEP 1005, 069 (2010) arXiv:1002.4633 [hep-ph]].

[9] L. Randall and R. Sundrum, Nucl. Phys. B 557 (1999) 79; G. Giudice, M. Luty, H. Murayama and R. Rattazzi, J. High Energy Phys. 12 (1998) 027.

[10] T. Gherghetta, G. Giudice and J. Wells, Nucl. Phys. B 559 (1999) 27.

[11] J. L. Feng, T. Moroi, L. Randall, M. Strassler and S. f. Su, Phys. Rev. Lett. 83 (1999) 1731 arXiv:hep-ph/9904250; J. L. Feng and T. Moroi, Phys. Rev. D 61 (2000) 095004; A. Datta and K. Huitu, Phys. Rev. D 67 (2003) 115006; S. Asai, T. Moroi, K. Nishihara and T. T. Yanagida, Phys. Lett. B 653 (2007) 81; S. Asai, T. Moroi and T. T. Yanagida, Phys. Lett. B 664 (2008) 185; H. Baer, J. K. Mizukoshi and X. Tata, Phys. Lett. B 488 (2000) 367; A. J. Barr, C. Lester, M. Parker, B. Allanach and P. Richardson, J. High Energy Phys. 0303 (2003) 045.

[12] LEPSUSYWG, note LEPSUSYWG/02-04.1.

[13] H. Baer, J. Ellis, G. Gelmini, D. V. Nanopoulos and X. Tata, Phys. Lett. B 161 (1985) 175; G. Gamberini, Z. Physik C 30 (1986) 605; H. Baer, V. Barger, D. Karatas and X. Tata, Phys. Rev. D 36 (1987) 96.

[14] R. Dermisek, H. Verlinde and L-T. Wang, Phys. Rev. Lett. 100 (2008) 131804; H. Baer. R. Dermisek, S. Rajagopalan and H. Summy, J. High Energy Phys. 0910 (2009) 078.

[15] H. Baer, R. Dermisek, S. Rajagopalan and H. Summy, JCAP1007 (2010) 014. 
[16] E. Komatsu et al. (WMAP collaboration), arXiv:1001.4538 (2010).

[17] T. Moroi and L. Randall, Nucl. Phys. B 570 (2000) 455; P. Grajek, G. Kane, D. Phalen, A. Pierce and S. Watson, Phys. Rev. D 79 (2009) 043506; G. Kane, R. Lu and S. Watson, Phys. Lett. B 681 (2009) 151; D. Feldman, G. Kane, R. Lu and B. Nelson, Phys. Lett. B 687 (2010) 363.

[18] K. Kohri, M. Yamaguchi and J. Yokoyama, Phys. Rev. D 72 (2005) 083510; T. Asaka, S. Nakamura and M. Yamaguchi, Phys. Rev. D 74 (2006) 023520; M. Endo, F. Takahashi and T. T. Yanagida, Phys. Rev. D 76 (2007) 083509.

[19] K.-Y. Choi, J. E. Kim, H. M. Lee and O. Seto, Phys. Rev. D 77 (2008) 123501.

[20] V. S. Kaplunovsky and J. Louis, Phys. Lett. B 306, 269 (1993) arXiv:hep-th/9303040. V. Kaplunovsky and J. Louis, Nucl. Phys. B 422 (1994) 57 [arXiv:hep-th/9402005]. S. P. de Alwis, Phys. Rev. D 77 (2008) 105020 arXiv:0801.0578.

[21] S. P. de Alwis "Classical and Quantum SUSY Breaking Effects in IIB Local Models," arXiv:0912.2950.

[22] D. E. Kaplan, G. D. Kribs and M. Schmaltz, Phys. Rev. D 62 (2000) 035010; Z. Chacko, M. A. Luty, A. E. Nelson and E. Ponton, J. High Energy Phys. 01 (2000) 003; for phenomenology, see M. Schmaltz and W. Skiba, Phys. Rev. D 62 (2000) 095005 and Phys. Rev. D 62 (2000) 095004; H. Baer, A. Belyaev, T. Krupovnickas and X. Tata, Phys. Rev. D 65 (2002) 075024; A. De Simone, J. J. Fan, M. Schmaltz and W. Skiba, Phys. Rev. D 78 (2008) 095010.

[23] J. R. Ellis, C. Kounnas and D. V. Nanopoulos, Nucl. Phys. B 247 (1984) 373; J. R. Ellis, A. B. Lahanas, D. V. Nanopoulos and K. Tamvakis, Phys. Lett. B 134 (1984) 429; G. A. Diamandis, J. R. Ellis, A. B. Lahanas and D. V. Nanopoulos, Phys. Lett. B 173 (1986) 303; For a review, see A. Lahanas and D. V. Nanopoulos, Phys. Rept. 145 (1987) 1.

[24] V. Balasubramanian, P. Berglund, J. P. Conlon and F. Quevedo, J. High Energy Phys. 03 (2005) 007.

[25] M. Grana, Phys. Rept. 423, 91 (2006) arXiv:hep-th/0509003.

[26] F. Paige, S. Protopopescu, H. Baer and X. Tata, hep-ph/0312045.

[27] H. E. Haber, R. Hempfling and A. Hoang, Z. Physik C 75 (1997) 539.

[28] D. Pierce, J. Bagger, K. Matchev and R. Zhang, Nucl. Phys. B 491 (1997) 3.

[29] G. Belanger, S. Kraml and A. Pukhov, Phys. Rev. D 72 (2005) 015003.

[30] H. Baer, B. Harris and M. H. Reno, Phys. Rev. D 57 (1998) 5871.

[31] W. Beenakker, M. Klasen, M. Kramer, T. Plehn, M. Spira and P. M. Zerwas, Phys. Rev. Lett. 83 (1999) 3780 [Erratum-ibid. 100 (2008) 029901] arXiv:hep-ph/9906298.

[32] Prospino, by W. Beenakker, R. Hopker and M. Spira, arXiv:hep-ph/9611232. 
[33] G. Bozzi, B. Fuks and M. Klasen, Phys. Lett. B 609 (2005) 339, Phys. Rev. D 74 (2006) 015001, Nucl. Phys. B 777 (2007) 157 and Nucl. Phys. B 794 (2008) 46.

[34] H. Baer, A. Bartl, D. Karatas, W. Majerotto and X. Tata, Int. J. Mod. Phys. A 4 (1989) 4111.

[35] G. Corcella et al. (Herwig collaboration), hep-ph/0210213.

[36] E. Richter-Was, arXiv:hep-ph/0207355.

[37] V. M. Abazov et al. (D0 Collaboration), Phys. Rev. Lett. 103 (2009) 141801.

[38] T. Aaltonen et al. (CDF Collaboration), Phys. Rev. D 77 (2008) 112001.

[39] T. Aaltonen et al. (CDF Collaboration), Phys. Rev. Lett. 99 (2007) 052002.

[40] W. L. van Neerven, J. A. M. Vermaseren and K. Gaemers, W. L. van Neerven, J. A. M. Vermaseren and K. J. F. Gaemers, NIKHEF-H/82-20 (1982); J. Smith, W. L. van Neerven and J. A. M. Vermaseren, Phys. Rev. Lett. 50 (1983) 1738; V. Barger, A. D. Martin and R. J. N. Phillips, Z. Physik C 21 (1983) 99

[41] C. G. Lester and D. J. Summers, Phys. Lett. B 463 (1999) 99; A. Barr, C. Lester and P. Stephens, J. Phys. G 29, 2343 (2003) arXiv:hep-ph/0304226]; A. J. Barr and C. G. Lester, arXiv:1004.2732 [hep-ph].

[42] H. Baer, V. Barger, A. Lessa and X. Tata, J. High Energy Phys. 1006 (2010) 102. 